\title{
Long-Term Efficacy of Olmesartan Medoxomil in Chinese Hypertensive Patients as Assessed by Clinic, Ambulatory and Home Blood Pressure Measurements
}

\author{
Ji-Guang Wang $\cdot$ Ning-Ling Sun $\cdot$ Yuan-Nan Ke $\cdot$ \\ Bo-Heng Zhang $\cdot$ Naotaka Ikegami $\cdot$ Jun-Ren Zhu
}

Published online: 7 September 2012

(c) The Author(s) 2012. This article is published with open access at Springerlink.com

\begin{abstract}
Background and Objectives There is limited information on the long-term efficacy and safety of olmesartan medoxomil in the management of hypertension in Chinese patients. We therefore conducted the present multicentre, single-arm, prospective, observational study to investigate the 24-week efficacy and safety of olmesartan medoxomil in patients with mild to moderate hypertension.

Methods Eligible patients (diastolic blood pressure [BP] 90-109 mmHg and systolic BP $<180 \mathrm{mmHg}$ off antihypertensive medication) were started on olmesartan medoxomil $20 \mathrm{mg}$ once daily, with the possible up-titration
\end{abstract}

J.-G. Wang ( $\bowtie)$

Centre for Epidemiological Studies and Clinical Trials,

The Shanghai Institute of Hypertension, Ruijin Hospital,

Shanghai Jiaotong University School of Medicine,

Ruijin 2nd Road 197, 200025 Shanghai, China

e-mail: jiguangw@gmail.com

N.-L. Sun

Department of Cardiology, People's Hospital,

Peking University, Beijing, China

Y.-N. Ke

Department of Cardiology, China-Japan Friendship Hospital,

Beijing, China

B.-H. Zhang

Division of Scientific Research, Zhongshan Hospital,

Fudan University, Shanghai, China

N. Ikegami

R\&D Division, Daiichi Sankyo Pharmaceutical

(Shanghai) Co., Ltd, Shanghai, China

J.-R. Zhu

Department of Cardiology, Zhongshan Hospital,

Fudan University, Shanghai, China to $40 \mathrm{mg}$ once daily during 24 weeks of follow-up, to control clinic BP to the target level $(<140 / 90$ and $<130 / 80 \mathrm{mmHg}$ in diabetes mellitus). In a subset of enrolled patients, 24-h ambulatory and home BP monitoring were also performed.

Results In the intent-to-treat analysis $(n=348)$, at 24 weeks of follow-up, the mean \pm SD changes from baseline in clinic systolic/diastolic BP were $21.2 \pm 14.2$ / $16.0 \pm 8.8 \mathrm{mmHg}(p<0.001)$. The proportions of patients who achieved the goal BP for systolic, diastolic and both were 81,80 and $75 \%$, respectively. Olmesartan medoxomil also significantly $(p<0.001)$ reduced systolic/diastolic BP measured at patients' homes by $17.7 \pm 13.1 / 12.1 \pm$ $7.9 \mathrm{mmHg}$ from baseline $(n=109)$, and reduced mean 24-h, daytime and night-time ambulatory BP by $13.3 \pm$ $16.3 / 7.6 \pm 9.5 \mathrm{mmHg}, \quad 13.9 \pm 17.4 / 8.0 \pm 10.4 \mathrm{mmHg}$ and $12.3 \pm 18.1 / 6.8 \pm 10.2 \mathrm{mmHg}$, respectively $(n=87)$. Seven $(2.0 \%)$ serious adverse events were reported during follow-up.

Conclusion In Chinese hypertensive patients, olmesartan medoxomil is efficacious in lowering BP as assessed by three different BP-measuring methods and has an acceptable long-term safety and tolerability profile.

\section{Introduction}

Olmesartan medoxomil is one of the most recently developed angiotensin II type 1 receptor antagonists (angiotensin receptor blockers [ARBs]), and has been available in the Chinese market for a few years [1]. Previous studies in American [2] and European populations [3] have demonstrated that olmesartan medoxomil is more efficacious in reducing blood pressure (BP) than other ARBs at equivalent dosages. Indeed, in a multicentre, randomized, doubleblind, 8-week, comparative trial of four ARBs, the mean 
reduction in clinic diastolic $\mathrm{BP}$ (DBP) from baseline was significantly greater with olmesartan medoxomil $20 \mathrm{mg}$ daily $(11.5 \mathrm{mmHg})$ than with losartan $50 \mathrm{mg}$ daily $(8.2 \mathrm{mmHg})$, valsartan $80 \mathrm{mg}$ daily $(7.9 \mathrm{mmHg})$ and irbesartan $150 \mathrm{mg}$ daily $(9.9 \mathrm{mmHg})$ [2]. Similar findings were observed in the same study for clinic systolic BP (SBP) [2] and for ambulatory BP [4], and in a meta-analysis of 36 studies that compared various ARBs with each other or with other classes of antihypertensive drugs or placebo [5].

However, there is still very limited information on the long-term efficacy and safety of olmesartan medoxomil in the management of hypertension in Chinese patients. We therefore conducted the present multicentre, single-arm, prospective, observational study to investigate the 24 -week efficacy and safety of olmesartan medoxomil 20-40 mg once daily in treating mild to moderate hypertension in Chinese patients.

\section{Methods}

\subsection{Study Design}

This multicentre, open-label, single-arm, prospective study was conducted in the outpatient clinic of 16 tertiary hospitals in China. The study protocol was approved by the Ethics Committee of Zhongshan Hospital, Fudan University, Shanghai, China, and as appropriate also by the Ethics Committees of the participating hospitals. All subjects gave written informed consent.

\subsection{Patients}

Patients eligible for inclusion in the present study had to be aged 18-75 years, and have a baseline clinic DBP of 90-109 mmHg and a clinic SBP below $180 \mathrm{mmHg}$, after being off antihypertensive medication for at least 1 week. Clinic BP was an average of six readings taken at two runin clinic visits, at which BP was measured on the right arm three times consecutively by use of a standard mercury sphygmomanometer. Exclusion criteria were secondary hypertension, isolated systolic hypertension (clinic SBP $\geq 140 \mathrm{mmHg}$ and clinic DBP $<90 \mathrm{mmHg}$ ), obesity (body mass index $\geq 30 \mathrm{~kg} / \mathrm{m}^{2}$ or body weight $\geq 100 \mathrm{~kg}$ ), use of $\beta$-blockers or agents that would influence BP, pregnancy or childbearing potential, severe liver (serum alanine transaminase $\geq 2$ times the upper limit of the normal range) and renal (serum creatinine $\geq 1.5$ times of the upper limit of the normal range or proteinuria $\geq 2+$ on a dipstick test) function impairment, indications for using other drugs that may affect the BP of patients, hypersensitivity to the study drug, and other conditions that the investigator thought inappropriate for study enrolment.

\subsection{Treatment and Follow-Up}

All enrolled subjects were treated initially with olmesartan medoxomil $20 \mathrm{mg}$ once daily, with the possible up-titration to $40 \mathrm{mg}$ once daily at 4, 8, 12, 16 and 20 weeks of followup, to achieve the goal of clinic SBP/DBP control to a level of $\leq 130 / 80 \mathrm{mmHg}$ in diabetes mellitus or $\leq 140 / 90 \mathrm{mmHg}$ in the absence of diabetes. Other antihypertensive drugs could be added if clinic BP exceeded $180 \mathrm{mmHg}$ SBP or $110 \mathrm{mmHg}$ DBP. Olmesartan medoxomil was supplied free by Daiichi Sankyo Pharmaceutical (Shanghai) Co., Ltd for the whole study period, and as instructed was taken in the early morning hours after getting up.

Clinic BP was measured on the right arm three times consecutively by the investigators using a standard mercury sphygmomanometer immediately before olmesartan medoxomil was taken. BP was also measured in a similar fashion at the subjects' home for 7 consecutive days before each of the clinic visits using an automated electronic BP monitor (HEM-4021, Omron, Kyoto, Japan). In all subjects from five of the 16 participating hospitals that consented, ambulatory BP monitoring was performed at baseline and at 24 weeks of follow-up using a validated BP monitor (SpaceLabs 90207 and 90217, SpaceLabs Healthcare, Issaquah, WA, USA). On the day of ambulatory BP monitoring, olmesartan medoxomil was taken after ambulatory BP monitoring was started.

\subsection{Efficacy and Safety Evaluations}

Efficacy was primarily evaluated as the changes from baseline in clinic SBP and DBP measured immediately before the study drug was taken at 24 weeks of follow-up. The secondary efficacy variables included clinic BP changes from baseline to 4, 8, 12, 16 and 20 weeks of follow-up, the proportion of patients who attained the goal clinic BP and, as appropriate, the BP changes from baseline for ambulatory and home monitoring during follow-up.

All adverse events reported by patients or observed by investigators at any time during the trial were recorded on a case report form and assessed for seriousness and relationship to the study drug. The results of all laboratory tests were also assessed by investigators for clinical significance and for possible relationship to the study drug.

\subsection{Statistical Methods}

For efficacy, we performed an intent-to-treat analysis in patients who complied with all the required criteria for inclusion and who started treatment with olmesartan 
medoxomil and had at least one follow-up visit; a perprotocol analysis was performed in patients who completed the 24-week follow-up. Categorical and continuous variables were analysed by the Chi-squared $\left(\chi^{2}\right)$ test and analysis of variance (ANOVA), respectively. The changes in BP from baseline to various follow-up visits were analysed with the paired $t$ test. The safety analysis included all enrolled patients who had started treatment with olmesar$\tan$ medoxomil. Adverse event data were analysed by Fisher's exact test. A $p$ value $\leq 0.05$ was considered statistically significant.

\section{Results}

\subsection{Characteristics of Patients}

Of the 360 patients enrolled in the present study, 357 had started treatment with olmesartan medoxomil and were therefore included in the safety analysis. Of these 357 patients, nine were excluded from intent-to-treat analysis because they did not fully comply with all the study requirements as defined in the study protocol. Of these 348 patients, 46 were further excluded from the per-protocol analysis because they were lost to follow-up $(n=7)$, had added other antihypertensive drugs for uncontrolled BP $(n=20)$ or because they withdrew from the study medication for adverse events $(n=12)$ or other reasons $(n=7)$. Thus, the intent-to-treat and per-protocol analyses included 348 and 302 patients, respectively (Fig. 1).

The 357 patients in the safety analysis comprised 164 women $(45.9 \%)$ and $25(7.0 \%)$ patients with diabetes at baseline. Mean \pm SD values at baseline were $52.2 \pm 9.0$ years of age, $24.9 \pm 2.8 \mathrm{~kg} / \mathrm{m}^{2}$ for body mass index and $149.0 \pm 11.0 \mathrm{mmHg} / 97.2 \pm 5.0 \mathrm{mmHg}$ for clinic SBP/ DBP (Table 1).

During follow-up, of the 348 patients in the intent-totreat analysis, $177(50.9 \%)$ remained on olmesartan medoxomil $20 \mathrm{mg}$ throughout the 24 weeks of follow-up, $135(38.8 \%)$ up-titrated to $40 \mathrm{mg}$ daily at $4-20$ weeks of

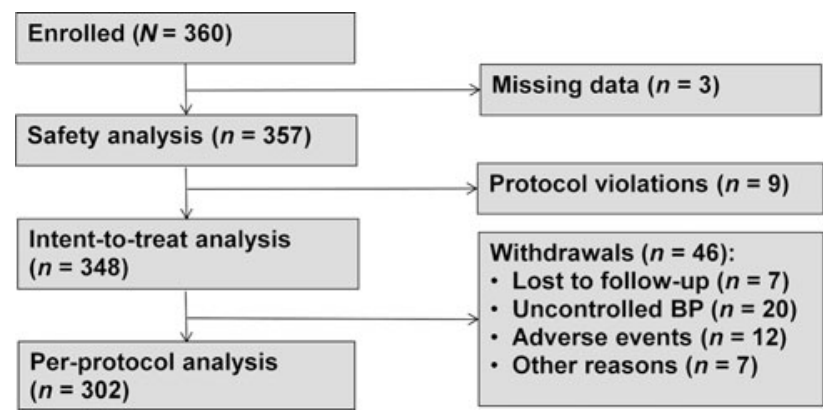

Fig. 1 Patient flow chart. $B P$ blood pressure
Table 1 Patient characteristics at baseline $(n=357)$

\begin{tabular}{llll}
\hline Characteristic & $\begin{array}{l}\text { Men } \\
(n=193)\end{array}$ & $\begin{array}{l}\text { Women } \\
(n=164)\end{array}$ & $p$ Value \\
\hline Age (years) & $51.2 \pm 9.6$ & $54.0 \pm 8.4$ & 0.005 \\
$\begin{array}{l}\text { Body mass index }\left(\mathrm{kg} / \mathrm{m}^{2}\right) \\
\text { Blood pressure }(\mathrm{mmHg})\end{array}$ & $25.4 \pm 2.6$ & $24.7 \pm 3.1$ & 0.02 \\
$\quad$ Clinic systolic & $147.8 \pm 12.0$ & $150.3 \pm 10.9$ & 0.05 \\
Clinic diastolic & $97.6 \pm 5.1$ & $96.8 \pm 4.9$ & 0.16 \\
Heart rate (beats/min) & $74.0 \pm 9.1$ & $73.9 \pm 8.6$ & 0.93 \\
Diabetes mellitus & $13(6.7 \%)$ & $12(7.3 \%)$ & 0.76 \\
\hline
\end{tabular}

Values are mean \pm SD except for diabetes mellitus ( $n[\%]$ ), which was defined as a fasting plasma glucose concentration of at least $7.1 \mathrm{mmol} / \mathrm{L}$ or the use of antidiabetic drugs

follow-up, and $36(10.3 \%)$ stopped olmesartan medoxomil with or without the addition of other antihypertensive drugs.

\subsection{Blood Pressure Reductions on Clinic Measurements}

In the intent-to-treat analysis $(n=348)$, at 24 weeks of follow-up or the last follow-up visit, the mean $\pm \mathrm{SD}$ changes in clinic SBP/DBP from baseline were $21.2 \pm$ $14.2 / 16.0 \pm 8.8 \mathrm{mmHg}$ ( $p<0.001$, Fig. 2$)$, and the proportions of patients who achieved the BP target for SBP, DBP and both were 81, 80 and $75 \%$, respectively (Fig. 3). At $4,8,12,16$ and 20 weeks of follow-up, the mean \pm SD changes from baseline in SBP/DBP were $15.3 \pm 14.2 /$ $11.8 \pm 9.0 \mathrm{mmHg}, 19.0 \pm 14.1 / 14.7 \pm 8.8 \mathrm{mmHg}, 20.1 \pm$ $13.8 / 15.3 \pm 8.4 \mathrm{mmHg}, \quad 21.1 \pm 14.5 / 15.7 \pm 9.0 \mathrm{mmHg}$ and $21.5 \pm 14.4 / 16.2 \pm 8.8 \mathrm{mmHg}$, respectively $(p<0.001$, Fig. 2).

Similar findings were observed in the per-protocol analysis $(n=302)$, with slightly higher proportions of patients who achieved the goal BP for SBP (82\%), DBP (82 \%) and both (77\%) at 24 weeks of follow-up (Figs. 2 and 3).

\subsection{Home and Ambulatory Blood Pressure Monitoring}

In 109 patients, BP was measured at the subjects' home. The mean $\pm \mathrm{SD}$ changes from baseline in SBP/DBP were $11.4 \pm 12.5 / 8.9 \pm 7.7 \mathrm{mmHg}, 13.9 \pm 11.8 / 9.9 \pm 7.7$ $\mathrm{mmHg}, \quad 15.5 \pm 12.1 / 10.6 \pm 8.0 \mathrm{mmHg}, \quad 16.1 \pm 12.1 /$ $11.3 \pm 7.8 \mathrm{mmHg}, 17.6 \pm 12.7 / 12.1 \pm 7.8 \mathrm{mmHg}$ and $17.7 \pm 13.1 / 12.1 \pm 7.9 \mathrm{mmHg}$ at $4,8,12,16,20$ and 24 weeks of follow-up, respectively $(p<0.001)$.

In 87 patients, ambulatory BP monitoring was performed. The mean $\pm \mathrm{SD}$ changes from baseline to 24 weeks of follow-up were $13.3 \pm 16.3 / 7.6 \pm 9.5 \mathrm{mmHg}$, 
Fig. 2 Mean \pm SD changes from baseline in clinic and home SBP and DBP at 4, 8, 12, 16,20 and 24 weeks of followup in (a) the intent-to-treat $(n=348)$ and $(\mathbf{b})$ the perprotocol $(n=302)$ analyses. The differences between baseline and follow-up values were statistically significant for all follow-up visits $(p<0.001)$. $B P$ blood pressure; $D B P$ diastolic BP; $S B P$ systolic BP
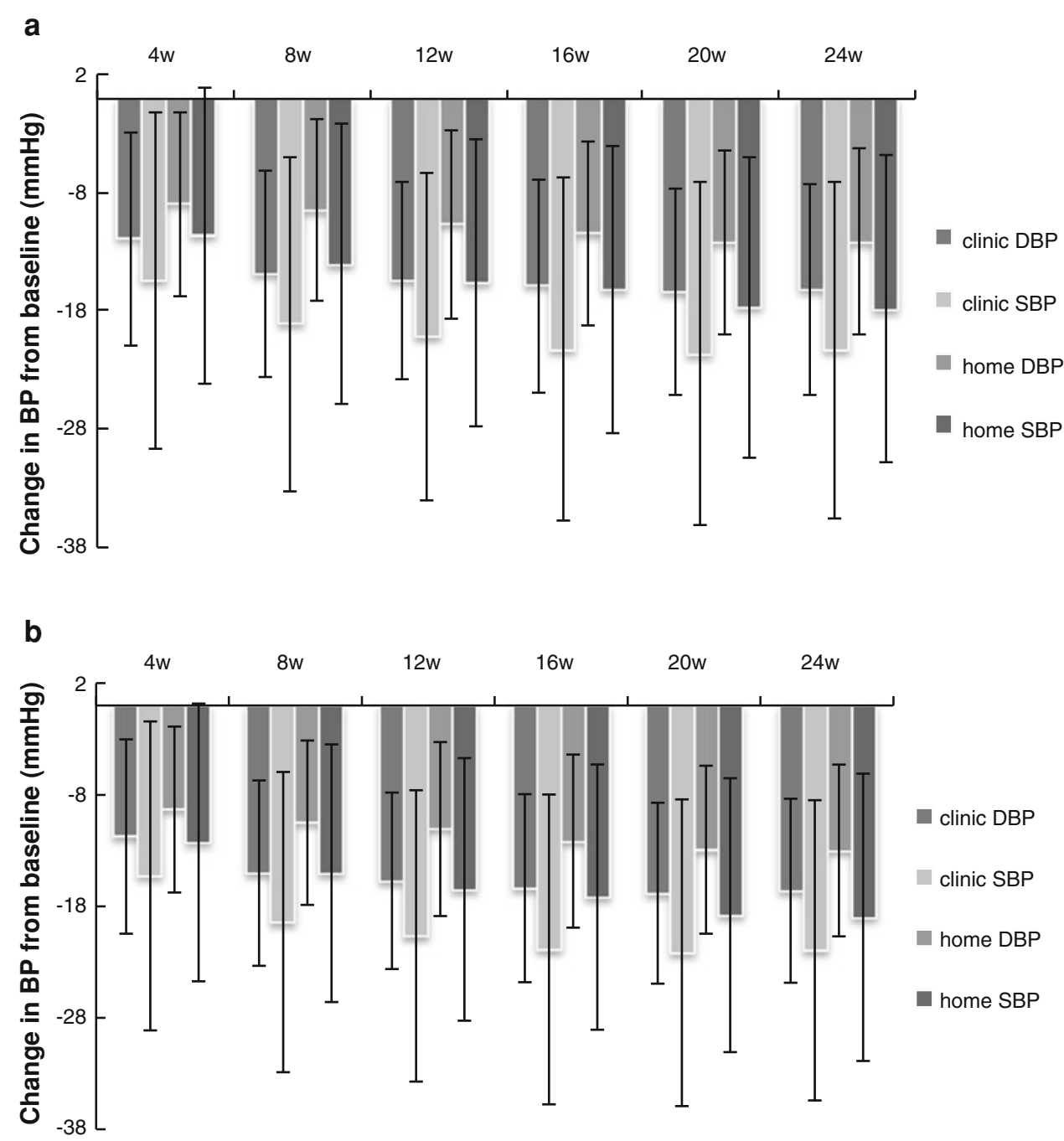

$13.9 \pm 17.4 / 8.0 \pm 10.4 \mathrm{mmHg}$ and $12.3 \pm 18.1 / 6.8 \pm 10.2$ $\mathrm{mmHg}$ for 24-h, daytime and night-time SBP/DBP, respectively $(p<0.001)$.

\subsection{Safety and Tolerability}

Of the 357 patients who had ever taken olmesartan medoxomil during follow-up, $80(22.4 \%)$ reported at least one episode of adverse event, including seven $(2.0 \%)$ patients with a serious adverse event (one for each of the following seven events: haemorrhagic stroke, myocardial infarction, unstable angina pectoris, glomerular nephritis, elevated serum concentration of alanine transaminase, dizziness and lumbar disc herniation) and 33 (9.2\%) patients with an adverse event that was considered by the investigator to be related to the use of the study drug (including one serious adverse event). The incidence rates of dizziness, upper respiratory tract infection, headache, asthenia, visual disturbance, flatulence and elevation of serum alanine transaminase exceeded $1 \%$ of the enrolled study participants (Table 2).

\section{Discussion}

Our study demonstrated similar BP reductions from baseline to several comparative studies that had a washout run-in phase and compared olmesartan medoxomil with placebo [6,7], other ARBs [1-3, 8, 9] or other classes of antihypertensive drugs [6,7] in American [2, 6, 7], Chinese $[1,8]$ and European populations [3,9].

In a multicentre, randomized, double-blind trial in patients with diastolic hypertension (a clinic DBP of $100-115 \mathrm{mmHg}$ and a mean daytime ambulatory DBP of $90-120 \mathrm{mmHg}$ ), the reductions in clinic SBP/DBP from baseline were $-11.3 /-11.5 \mathrm{mmHg}$ after 8 weeks of treatment with olmesartan medoxomil $20 \mathrm{mg}$ daily [2]. The corresponding values in mean daytime ambulatory blood 

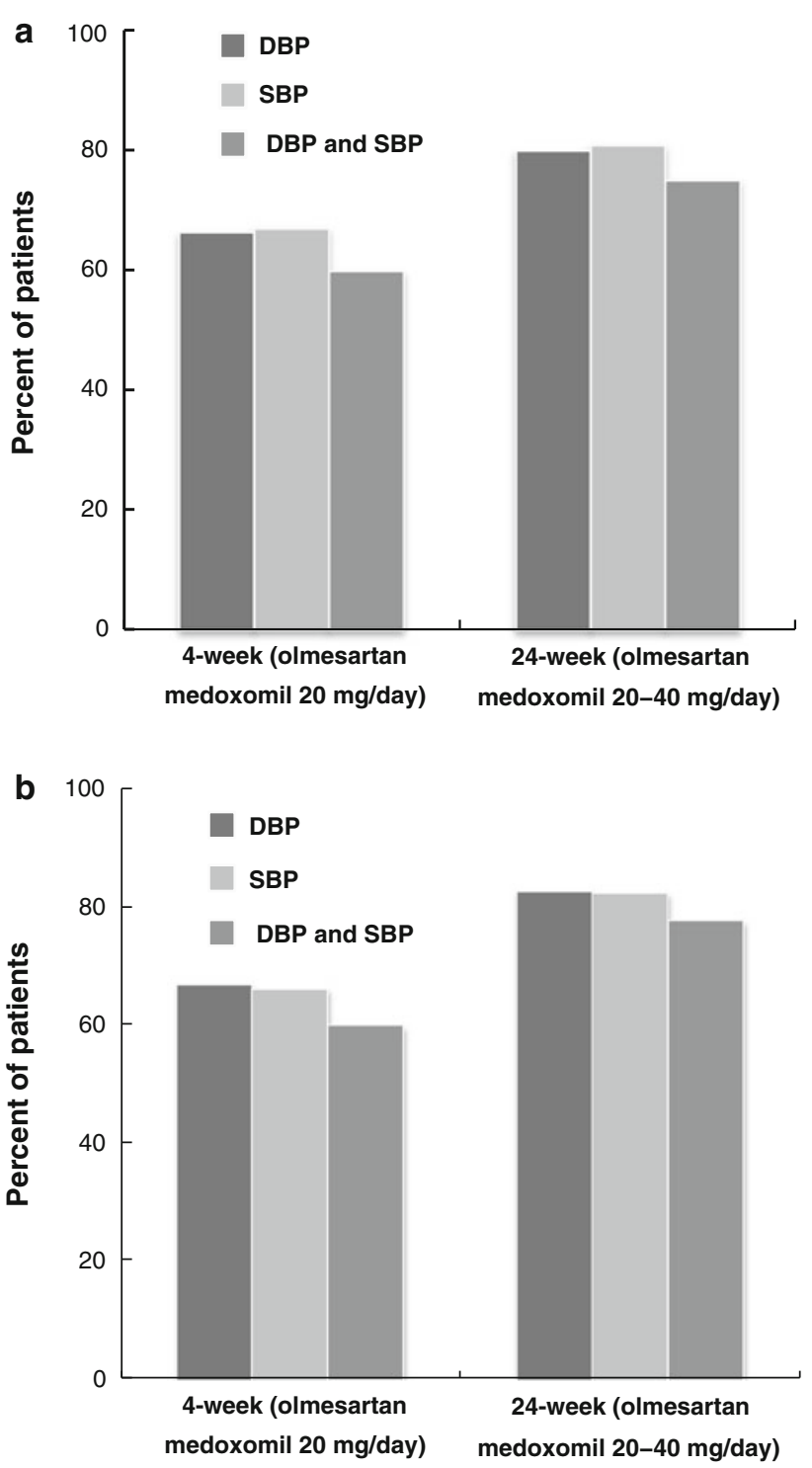

Fig. 3 Proportion of patients who attained the goal blood pressure for SBP $(<130 \mathrm{mmHg}$ in patients with diabetes mellitus or $<140 \mathrm{mmHg}$ in the absence of diabetes), DBP $(<80 \mathrm{mmHg}$ or $<90 \mathrm{mmHg}$, respectively), and both SBP and DBP at 4 weeks (olmesartan medoxomil $20 \mathrm{mg} /$ day) and 24 weeks (olmesartan medoxomil $20-40 \mathrm{mg} /$ day $)$ of follow-up in (a) the intent-to-treat $(n=348)$ and (b) the per-protocol $(n=302)$ analyses. $D B P$ diastolic blood pressure; $S B P$ systolic blood pressure

pressure were $-12.5 /-8.5 \mathrm{mmHg}$ [2]. In an 8-week, randomized, double-blind, parallel-group study, the mean changes from baseline to 8 weeks of follow-up in the olmesartan medoxomil ( $20 \mathrm{mg}$ daily) group were $-21.2 /$ $-15.8 \mathrm{mmHg}$ for clinic SBP/DBP and $-13.0 /-9.3 \mathrm{mmHg}$ for mean daytime ambulatory DBP [3]. In a 12-week, randomized, double-blind, forced-titration study, the mean changes from baseline to 8 weeks of follow-up in the olmesartan medoxomil (40 mg once daily) group were $-13.9 /-11.7 \mathrm{mmHg}$ for clinic SBP/DBP [4]. In a meta-
Table 2 Adverse events in the safety analysis ( $n=357$ patients)

\begin{tabular}{lll}
\hline Adverse event & No. of patients & $\begin{array}{l}\text { Incidence rate } \\
(\%)\end{array}$ \\
\hline Dizziness & 22 & 6.2 \\
Upper respiratory tract & 10 & 2.8 \\
$\quad$ infection & & \\
Headache & 9 & 2.5 \\
Asthenia & 5 & 1.4 \\
Visual disturbance & 4 & 1.1 \\
Flatulence & 4 & 1.1 \\
Alanine transaminase elevation & 4 & 1.1 \\
\hline
\end{tabular}

Only adverse events with an incidence rate of $1 \%$ or higher were listed

analysis of seven randomized, double-blind, placebo-controlled, dose-finding studies (treatment with olmesartan medoxomil $2.5-80 \mathrm{mg}$ for 6-52 weeks) in the American and European populations, olmesartan medoxomil $20 \mathrm{mg}$ per day was significantly effective in lowering BP. The mean changes in SBP/DBP from baseline to 8 weeks of treatment were $-11.3 /-11.5 \mathrm{mmHg}$ [10].

The results of our study can also be compared with those of other non-comparative, prospective, observational studies of other ARBs, such as the recently published INCLUSIVE (irbesartan/hydrochlorothiazide blood pressure reductions in diverse patient populations) trial [11]. The INCLUSIVE study was an 8-week, multicentre, prospective, open-label, single-arm study that evaluated the efficacy and safety of irbesartan/hydrochlorothiazide 150 $\mathrm{mg} / 12.5 \mathrm{mg}$ to $300 \mathrm{mg} / 25 \mathrm{mg}$ in patients with uncontrolled SBP on monotherapy $(130-159 \mathrm{mmHg}$ in patients with diabetes and $140-159 \mathrm{mmHg}$ in the absence of diabetes). In the INCLUSIVE trial, the mean changes in clinic SBP/DBP were $-21.5 /-10.4 \mathrm{mmHg}$, and 77,83 and $69 \%$ of patients achieved the goal BP for SBP $(<130 \mathrm{mmHg}$ in patients with diabetes and $<140 \mathrm{mmHg}$ in the absence of diabetes), DBP ( $<80$ and $<90 \mathrm{mmHg}$ ) and both.

In keeping with the results of several previous studies $[2,3,12]$, our study demonstrated that BP reductions on clinic measurements were much larger than on daytime ambulatory monitoring (21.2/16.0 vs. $13.9 / 8.0 \mathrm{mmHg}$ at 24 weeks of follow-up). If home monitoring was compared with daytime ambulatory BP monitoring, the BP-lowering effects were also significantly greater with the former $(17.7 / 12.1 \mathrm{mmHg})$ than with the latter measuring techniques. These results suggest that the three different BPmeasuring methods might measure different BPs and hence have different clinical significances.

A major limitation of our study is its non-comparative design. Without a proper control group, placebo effects, observer bias and regression-to-the mean may influence the evaluation of BP-lowering efficacy especially when 
assessed by clinic measurements. However, observations in non-comparative studies, such as the amplitude of changes in BP from baseline and the rate of target BP attainment, might be directly applicable in real life clinical practice. In addition, we performed home and ambulatory BP monitoring in a subset of enrolled study subjects. These more objective methods of BP measurement might help minimize potential sources of bias.

Several other limitations of our study are also noteworthy. First, ambulatory and home BP monitoring were only performed in a subset of the enrolled subjects. Second, when the present trial was initiated, guidelines for home BP monitoring had not yet been published [13, 14]. In a similar fashion to clinic BP measurement, BP at home was measured only once immediately before olmesartan medoxomil was taken. Third, our study excluded patients with isolated systolic hypertension for practical operational reasons. The results of our study hence cannot be extrapolated to this common form of hypertension in the elderly.

\section{Conclusion}

In mild to moderate hypertension, about two-thirds of patients treated with olmesartan medoxomil $20 \mathrm{mg}$ daily may achieve the target BP after 4 weeks of treatment. With the longer term adherence to olmesartan medoxomil treatment and with the possible up-titration to $40 \mathrm{mg}$ daily, the rate of attaining goal BP may further increase to approximately $80 \%$. In Chinese hypertensive patients, olmesartan medoxomil appears to effectively lower BP regardless of BP measurement, and has an acceptable long-term safety and tolerability profile.

\begin{abstract}
Acknowledgments The authors gratefully acknowledge the participation of all study subjects. The principal investigators from the 16 participating hospitals (in alphabetical order) are: Nai-Sheng Cai (Zhongshan Hospital, Shanghai), Si-Yu Cai (The Second Affiliated Hospital of Zhejiang University of School of Medicine, Hangzhou), Yun-Dai Chen (The General Hospital of the People's Liberation Army, Beijing), Yu-Gang Dong (The First Affiliated Hospital of Sun Yat-Sen University, Guangzhou), Ben He (Renji Hospital, Shanghai), Hong-Wei Li (Friendship Hospital, Beijing), Yu-Hua Liao (Union Hospital, Wuhan), Guo-Xian Qi (The First Hospital of China Medical University, Shenyang), Hai-Ming Shi (Huashan Hospital, Shanghai), Dao-Wen Wang (Tongji Hospital, Wuhan), Hua Yao (Guangdong General Hospital, Guangzhou), Shu-Yang Zhang (Union Hospital, Beijing) and Jian-Hua Zhu (The First Affiliated Hospital of Zhejiang University School of Medicine, Hangzhou). The study was funded by Daiichi-Sankyo Pharmaceutical (Shanghai) Co. Ltd. Naokata Ikegami is an employee of Daiichi-Sankyo Pharmaceutical (Shanghai) Co. Ltd. Dr Wang reports receiving consulting and lecture fees from Daiichi-Sankyo Pharmaceutical (Shanghai) Co. Ltd. The other authors declare no conflicts of interest.
\end{abstract}

Open Access This article is distributed under the terms of the Creative Commons Attribution Noncommercial License which permits any noncommercial use, distribution, and reproduction in any medium, provided the original author(s) and source are credited.

\section{References}

1. Zhu JR, Cai NS, Fan WH, Zhu DL, He B, Wu ZG, Ke YN, Guo JX, Ma H, Huang J, Li XL, Chen YZ. Efficacy and safety of olmesartan medoxomil versus losartan potassium in Chinese patients with mild to moderate essential hypertension. Chin J Cardiol (in Chinese). 2006;34:877-81.

2. Oparil S, Williams D, Chrysant SG, Marbury TC, Neutel J. Comparative efficacy of olmesartan, losartan, valsartan, and irbesartan in the control of essential hypertension. J Clin Hypertens (Greenwich). 2001;3(283-91):318.

3. Brunner HR, Stumpe KO, Januszewicz A. Antihypertensive efficacy of olmesartan medoxomil and candesartan cilexetil assessed by 24-hour ambulatory blood pressure monitoring in patients with essential hypertension. Clin Drug Investig. 2003;23:419-30.

4. Smith DH, Dubiel R, Jones M. Use of 24-hour ambulatory blood pressure monitoring to assess antihypertensive efficacy: a comparison of olmesartan medoxomil, losartan potassium, valsartan, and irbesartan. Am J Cardiovasc Drugs. 2005;5:41-50.

5. Fabia MJ, Abdilla N, Oltra R, Fernandez C, Redon J. Antihypertensive activity of angiotensin II AT1 receptor antagonists: a systematic review of studies with $24 \mathrm{~h}$ ambulatory blood pressure monitoring. J Hypertens. 2007;25:1327-36.

6. Chrysant SG, Marbury TC, Robinson TD. Antihypertensive efficacy and safety of olmesartan medoxomil compared with amlodipine for mild-to-moderate hypertension. J Hum Hypertens. 2003; 17:425-32.

7. Chrysant SG, Marbury TC, Silfani TN. Use of 24-h ambulatory blood pressure monitoring to assess blood pressure control: a comparison of olmesartan medoxomil and amlodipine besylate. Blood Press Monit. 2006;11:135-41.

8. Liau CS, Lee CM, Sheu SH, Ueng KC, Chien KL, Su TC, Lai WT, Lin MC, Lin CS, Lin CS. Efficacy and safety of olmesartan in the treatment of mild-to-moderate essential hypertension in Chinese patients. Clin Drug Investig. 2005;25:473-9.

9. Brunner HR, Arakawa K. Antihypertensive efficacy of olmesartan medoxomil and candesartan cilexetil in achieving 24-hour blood pressure reductions and ambulatory blood pressure goals. Clin Drug Investig. 2006;26:185-93.

10. Brunner HR. Clinical efficacy and tolerability of olmesartan. Clin Ther. 2004;26(Suppl A):A28-32.

11. Neutel JM, Saunders E, Bakris GL, Cushman WC, Ferdinand KC, Ofili EO, Sowers JR, INCLUSIVE Investigators. The efficacy and safety of low- and high-dose fixed combinations of irbesartan/hydrochlorothiazide in patients with uncontrolled systolic blood pressure on monotherapy: the INCLUSIVE trial. J Clin Hypertens (Greenwich). 2005;7:578-86.

12. Brunner HR. Olmesartan medoxomil: current status of its use in monotherapy. Vasc Health Risk Manag. 2006;2:327-40.

13. Parati G, Stergiou GS, Asmar R, Bilo G, de Leeuw P, Imai Y, Kario K, Lurbe E, Manolis A, Mengden T, O’Brien E, Ohkubo T, Padfield P, Palatini P, Pickering TG, Redon J, Revera M, Ruilope LM, Shennan A, Staessen JA, Tisler A, Waeber B, Zanchetti A, ESH Working Group on Blood Pressure Monitoring. European Society of Hypertension practice guidelines for home blood pressure monitoring. J Hum Hypertens. 2010;24:779-85.

14. Pickering TG, Miller NH, Ogedegbe G, Krakoff LR, Artinian NT, American Heart Association, American Society of Hypertension, Preventive Cardiovascular Nurses Association. Call to action on use and reimbursement for home blood pressure monitoring: a joint scientific statement from the American Heart Association, American Society Of Hypertension, and Preventive Cardiovascular Nurses Association. Hypertension. 2008;52:10-29. 